Supplementary Materials for

\title{
Intrinsic Low Thermal Conductivity and Phonon Renormalization Due to Strong Anharmonicity of Single-Crystal Tin Selenide
}

\author{
Joon Sang Kang, Huan Wu, Man Li, Yongjie Hu${ }^{*}$ \\ School of Engineering and Applied Science, University of California, Los Angeles (UCLA), \\ Los Angeles, CA 90095, United States. \\ *Corresponding email: yhu@seas.ucla.edu .
}




\section{S1. Synthesis of SnSe crystals}

High quality single-crystal SnSe was synthesized through a repeated multi-step chemical vapor transport process. Details of the chemical vapor transport setups and synthesize process to grow highquality single crystals can be found in our recent publications [S1,S2,S5]. Stoichiometric ratio of highpurity Tin (Sn) and selenium (Se) (99.999\% purity, from Alfa Aesar) were placed into a quartz tube. This tube was evacuated and flame sealed under high vacuum ( $10^{-5}$ torr $)$. A customized multi-zone reactor was set up with reaction temperatures of $1223 \mathrm{~K}$ and $1173 \mathrm{~K}$ for the hot and cold zones, respectively. Temperature changes were controlled with a very slow rate for over 12 hours. The reactor was held at the reaction temperatures for over 96 hours and then cooled down to room temperature. The initial growth led to crystal ingots. The as-grown crystals were pulverized and returned to the quartz tube, which was evacuated, flame sealed, and loaded into the reactor. This growth procedure was repeated until high quality single crystal SnSe with the close-to-theory maximum density were obtained.

\section{S2. Nanoscale optic-thermal measurement}

Measurement of the intrinsic thermal conductivity of single crystal SnSe was performed using the time-domain thermoreflectance (TDTR), which has become a standard technique to measure the thermal conductivity of solids [S1-S8]. TDTR is well suited to study the intrinsic thermal conductivity of SnSe samples because no physical contact with the sample is required and the measurements can be performed over a region as small as $\sim 1$ micrometer in size. In the TDTR setup (Fig. S1a), a laser beam pump consisting of a train of short pulses ( 50 femtoseconds) created an instantaneous temperature rise at the sample surface. The transient temperature decay, from the heat impulse was monitored with another probe pulse that was delayed in time with respect to the pump using a mechanical delay stage (with subpicosecond resolution). The SnSe full transient decay curve was obtained by varying the time delay, and was subsequently fitted to a thermal model to deduce the thermal conductivity (Fig. S1c). In our setup 
(Fig. S1b), a Ti:Sapphire oscillator generated $800 \mathrm{~nm}$ wavelength, 50 100 femto-second pulse width, and $80 \mathrm{MHz}$ repetition rate laser pulses. The beam was split into pump and probe beams with a large power ratio. The pump beam frequency was sinusoidally modulated with an electric optic modulator (EOM) up to $20 \mathrm{MHz}$, and the fundamental frequency of the pump beam was doubled by bismuth triborate crystal (BIBO). The probe beam was delayed with a mechanical delay stage from 0 to 6000 ps. Both pump and probe beams were focused on the sample surface with an objective lens and the reflected signal of the probe beam was detected by an Si photodiode (Thorlabs PDA36A) connected to a lock-in amplifier for exclusive signal detection of the modulation frequency of the pump.

Mathematically, the detected signal can be expressed as:

$$
Z\left(f_{0}\right)=\sum_{N=-\infty}^{N=\infty} H\left(f_{0}+N f_{s}\right) e^{i N f_{s} \tau}
$$

where $H(f)$ is the response frequency, $f_{s}$ the pulse repetition frequency, and $\tau$ the time delay between the pump and probe beams. The response frequency function can be solved with the thermal diffusion model. For our measurements on SnSe, a one-dimensional heat conduction model was solved in the frequency domain. Additional details can be found in the literature [S4]. Amplitude and phase changes indicate a temperature change of the metal film and are functions of the time delay. The thermal conductivity of SnSe samples was obtained by fitting the experimental data to this theoretical model. Fig. S1(c) shows a typical TDTR phase change result along the c-axis of SnSe with its best fit to the thermal diffusion model. By intentionally changing the thermal conductivity values by $\pm 10 \%$, two other phase change curves were plotted (dashed black lines). The distribution of all the experimental data within the narrow range between the two dashed curves demonstrates the high accuracy of our thermal conductivity measurements. A detailed description of our nanoscale optic-thermal measurement technique and anisotropic thermal measurement can be found in our recent papers [S1-S5, S8]. 
(a)

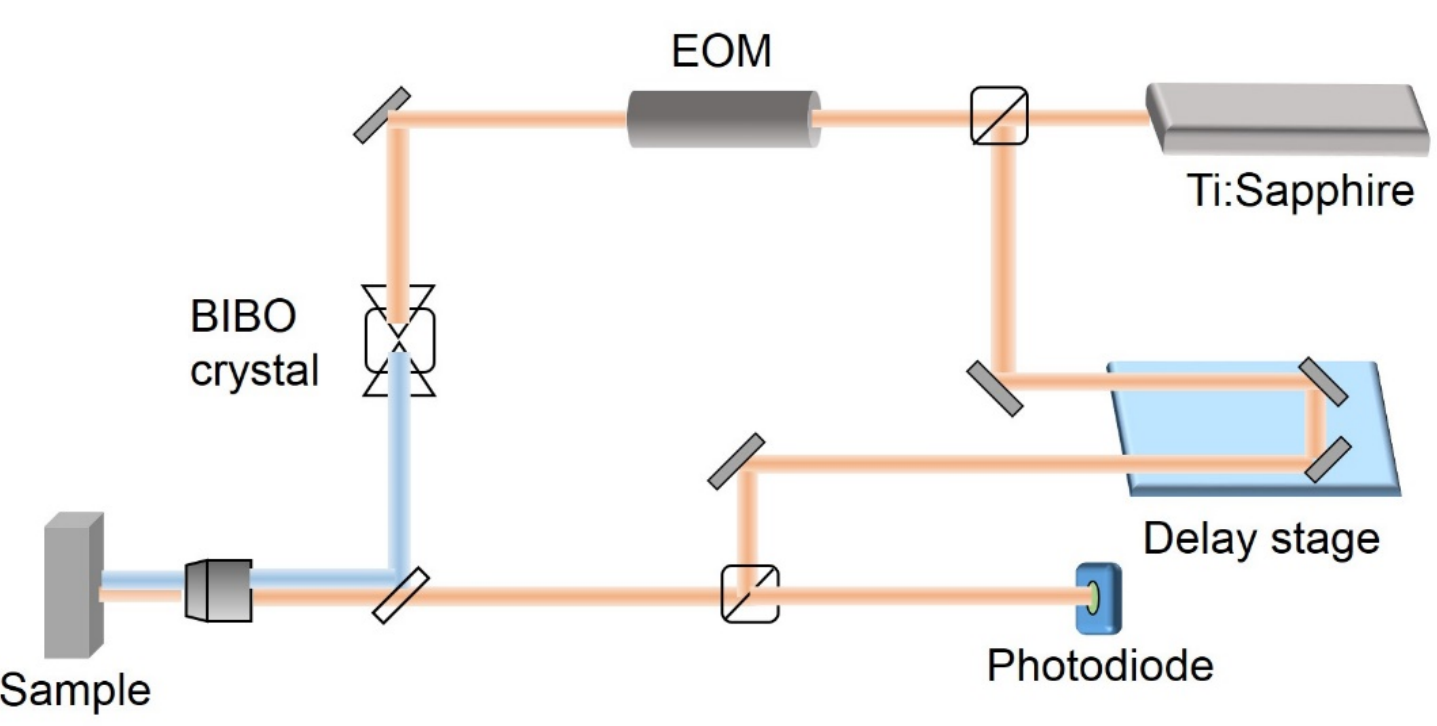

(b)

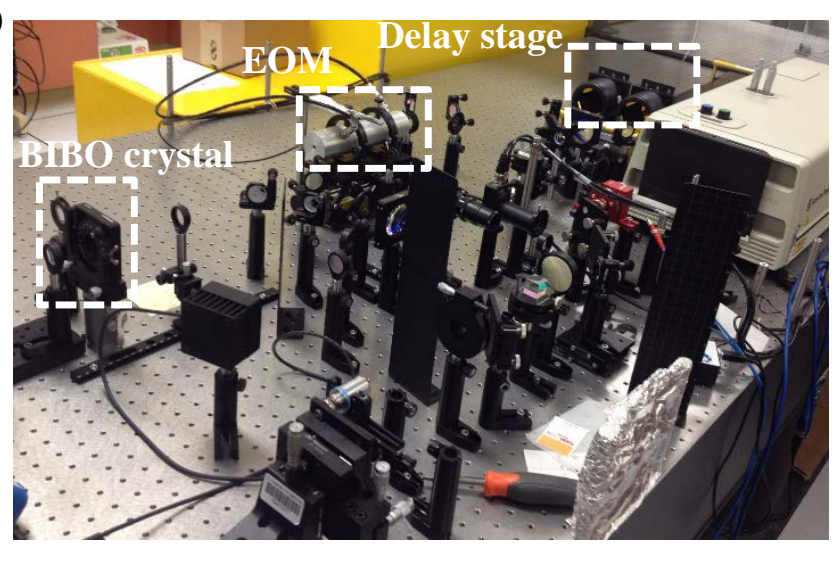

(c)

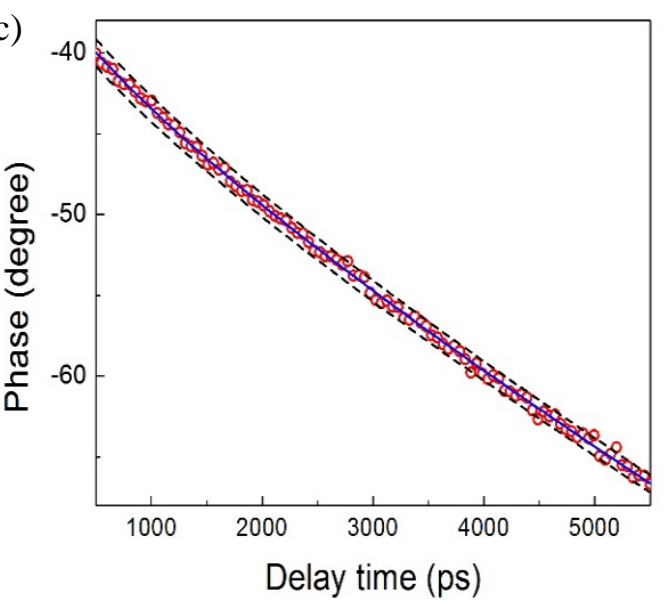

Figure S1. Time-domain thermoreflectance (TDTR) setup and a typical measurement on SnSe. (a) Schematic of the TDTR setup; (b) a photograph of the TDTR setup; (c) a typical TDTR measurement result (red circle, for the c-axis of SnSe), fitted to the thermal transport model (solid blue line). Plots of the calculated curves (black dashed lines), using the thermal conductivity changed by $\pm 10 \%$ of the best values, support a measurement uncertainty within $10 \%$.

\section{S3. Specific heat of SnSe}

The specific heat of SnSe was measured across the temperature range from 120 - $300 \mathrm{~K}$, using the differential scanning calorimetry (PerkinElmer DSC 8000), the two furnaces system and the StepScan mode of which ensured the measurement accuracy. The measurement parameters were set as $10 \mathrm{~K} / \mathrm{min}$ 
for the heating rate, $10 \mathrm{~K}$ for the temperature interval, $1 \mathrm{~min}$ for thermalization time and $20 \mathrm{~mL} / \mathrm{min}$ for the nitrogen purge flow rate. The temperature-dependent specific heat of SnSe was plotted by averaging ten repetitive measurement results (Fig. S3) [S10].

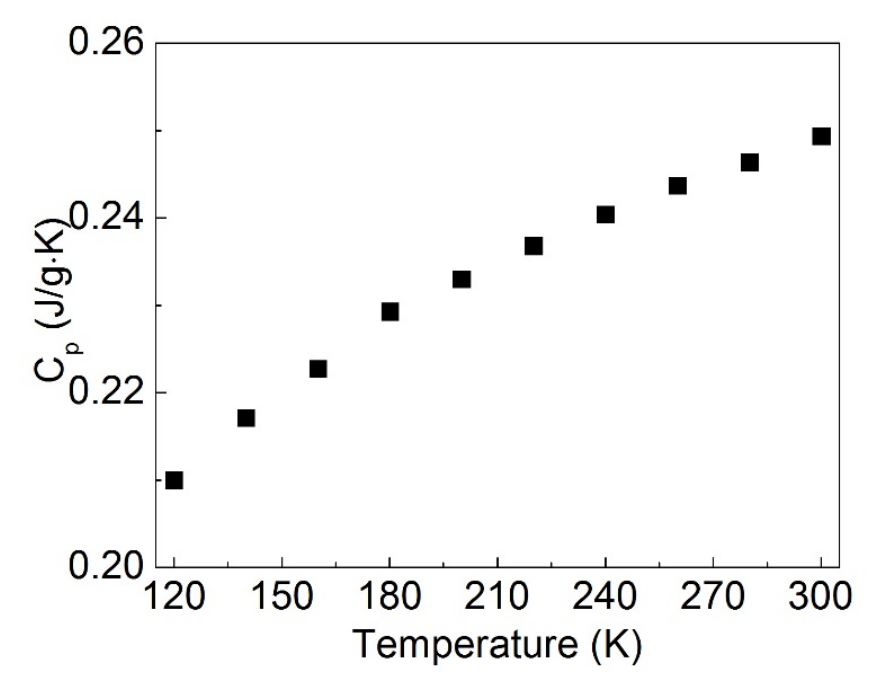

Figure S3. The temperature dependent specific heat of single-crystal SnSe.

\section{S4. Derivation of the Grüneisen parameter at the Brillouin zone center}

The phonon frequency $\left(\omega_{i}\right)$ depends on the material volume $(\mathrm{V})$ and the temperature $(\mathrm{T})$ $\omega_{i}(\mathrm{~V}, \mathrm{~T})$. The mode dependent Grüneisen parameter $\left(\gamma_{i}\right)$ and phonon group velocity $\left(v_{g i}\right)$ are defined by [S9]:

$$
\begin{aligned}
& \gamma_{i}=-\frac{V}{\omega_{i}} \frac{\partial \omega_{i}}{\partial V} \\
& v_{g i}=\frac{\partial \omega_{i}}{\partial \mathrm{q}_{\mathrm{i}}}
\end{aligned}
$$


where $q_{i}$ is the reciprocal vector along a specific crystal direction defined as $q_{i}=\frac{2 \pi i}{N * a_{i}} \quad$ ( $a_{i}$ is the lattice constant).

Near the Brillouin zone center, i.e., for small frequency phonons, $v_{g i}$ is independent of the frequency and is equivalent to the sound velocity $\left(v_{g i}\right)$,

$$
v_{g i}=v_{g}=\frac{\partial \omega_{i}}{\partial \mathrm{q}_{\mathrm{i}}}=\frac{\omega_{i}}{\mathrm{q}_{\mathrm{i}}}
$$

Under constant pressure,

$$
\begin{aligned}
& \frac{\partial \omega_{i}}{\partial T}=\frac{\partial\left(v_{g} * q_{i}\right)}{\partial T}=v_{g} * \frac{\partial\left(q_{i}\right)}{\partial T}+q_{i *} \frac{\partial\left(v_{g}\right)}{\partial T}=-v_{g} * \mathrm{q}_{\mathrm{i} *} \frac{\partial\left(a_{i}\right)}{a_{i} * \partial T}+q_{i} * \frac{\partial\left(v_{g}\right)}{\partial T} \\
& \frac{\partial \omega_{i}}{\omega_{i} * \partial T}=-v_{g} * \frac{\mathrm{q}_{\mathrm{i}}}{\omega_{i}} * \frac{\partial\left(a_{i}\right)}{a_{i} * \partial T}+\frac{\mathrm{q}_{\mathrm{i}}}{\omega_{i}} \frac{\partial\left(v_{g}\right)}{\partial T}=-\frac{\partial\left(a_{i}\right)}{a_{i} * \partial T}+\frac{1}{v_{g}} * \frac{\partial\left(v_{g}\right)}{\partial T}=-\alpha_{1}+\frac{1}{v_{g}} * \frac{\partial\left(v_{g}\right)}{\partial T}
\end{aligned}
$$

where $\alpha_{1}$ is the linear thermal expansion coefficient along the specific crystal orientation.

Therefore,

$$
\gamma_{i}=-\frac{V}{\omega_{i}} \frac{\partial \omega_{i}}{\partial V}=-\frac{\partial \omega_{i}}{\omega_{i} * \partial T} / \frac{\partial V}{V * \partial T}=-\frac{\partial \omega_{i}}{\omega_{i} * \partial T} / \alpha_{V}
$$

where $\alpha_{V}$ is the volumetric thermal expansion coefficient. From Equation (S6) and (S7), we have,

$$
\gamma_{i}=\frac{\alpha_{1}}{\alpha_{V}}-\frac{1}{\alpha_{V} * v_{g}} * \frac{\partial\left(v_{g}\right)}{\partial T}
$$

\section{S5. Ab initio calculations}

We performed $a b$ initio calculations of temperature dependent phonon dispersions of SnSe following three steps. First, density functional theory (DFT) was applied to find an optimized equilibrium structure with force acting on each ion less than $10^{-4} \mathrm{eV} / \AA$. Second, the second and fourth order IFCs were calculated by finite displacement method. We built a 144 atoms supercell, generated irreducible sets of displacement using ALAMODE package, calculated the force acting on each ion by DFT, and derived IFCs based that. For the $3^{\text {rd }}$ order IFCs, we consider up to $8^{\text {th }}$ nearest neighboring atoms for Sn atoms and up to $5^{\text {th }}$ nearest neighboring atoms for Se atoms. For $4^{\text {th }}$ order IFCs, we consider up to $4^{\text {th }}$ nearest 
neighboring atoms for both Sn atoms and Se atoms. The DFT calculations are accomplished using Quantum ESPRESSO[S11, S12]. For all the DFT calculations, we used projector augmented-wave pseudopotential with local density approximation [S13], and the electronic wave functions were expanded in plane-wave basis with kinetic energy cutoff at $544 \mathrm{eV}$. We used $4 \times 6 \times 6$ Monkhorst-Pack k-points meshes to sample the Brillouin zone for structure optimization, and $2 \times 2 \times 2$ Monkhorst-Pack k-points meshes for IFCs calculation. Third, we solved Eq. (8) using ALAMODE package[S14, S15] to get temperature dependent dispersion relation of SnSe.

\section{References}

S1. J. S. Kang, M. Li, H. Wu, H. Nguyen \& Y. Hu, Experimental observation of high thermal conductivity in boron arsenide. Science 361, 575-578 (2018).

S2. J.S. Kang, H. Wu, \& Y. Hu Thermal Properties and Phonon Spectral Characterization of Synthetic Boron Phosphide for High Thermal Conductivity Applications. Nano Lett. 17, 7507-7514 (2017).

S3. J.S. Kang, M. Ke, \& Y. Hu Ionic Intercalation in Two-Dimensional van der Waals Materials: In Situ Characterization and Electrochemical Control of the Anisotropic Thermal Conductivity of Black Phosphorus. Nano Lett. 17, 1431-1438 (2017).

S4. M. Li, J.S. Kang, and Y. Hu, "Anisotropic thermal conductivity measurement using a new Asymmetric-Beam Time-Domain Thermoreflectance (AB-TDTR) method," Review of Scientific Instruments 89, 084901 (2018).

S5. M. Li et al., Anisotropic Thermal Boundary Resistance across 2D Black Phosphorus: Experiment and Atomistic Modeling of Interfacial Energy Transport, Adv. Mater., 1901021 (2019).

S6. M. N. Luckyanova et al., Coherent Phonon Heat Conduction in Superlattices. Science. 338, 936939 (2012).

S7. C. Chiritescu et al., Ultralow Thermal Conductivity in Disordered, Layered WSe2 Crystals, Science, 315, 351-353 (2007).

S8. Y. Hu et al., Spectral mapping of thermal conductivity through nanoscale ballistic transport. Nature Nanotechnology 10, 701-706 (2015).

S9. J. M. Ziman, Principles of the theory of solids, Cambridge University Press, second edition, 1972.

S10. Differential Scanning Calorimetry by Günther Höhne, Wolfgang F. Hemminger, H.-J. Flammersheim. Springer, 2nd edition, 2010.

S11. Wentzcovitch, P. G. and S. B. and N. B. and M. C. and R. C. and C. C. and D. C. and G. L. C. and M. C. and I. D. and A. D. C. and S. de G. and S. F. QUANTUM ESPRESSO: a modular and open- 
source software project for quantum simulations of materials. J. Phys. Condens. Matter 21, 395502 (2009).

S12. Baroni, P. G. and O. A. and T. B. and O. B. and M. B. N. and M. C. and R. C. and C. C. and D. C. and M. C. and N. C. and I. C. and A. D. C. and S. de G. and P. D. and R. A. D. J. and A. Advanced capabilities for materials modelling with Quantum ESPRESSO. J. Phys. Condens. Matter 29, 465901 (2017).

S13. We used the pseudopotentials Sn.pz-dn-kjpaw_psl.0.2.UPF and Se.pz-n-kjpaw_psl.0.2.UPF from http://www.quantum-espresso.org.

S14. Tadano, T. \& Tsuneyuki, S. Self-consistent phonon calculations of lattice dynamical properties in cubic SrTiO with first-principles anharmonic force constants. Phys. Rev. B 92, 54301 (2015).

S15. Tadano, T., Gohda, Y., and Tsuneyuki, S. Anharmonic force constants extracted from firstprinciples molecular dynamics: applications to heat transfer simulations. J. Phys.: Condens. Matter 26, 225402 (2014). 